\title{
Arithmetic and Matricial Calculation
}

\section{Raoelina Andriambololona, Ravo Tokiniaina Ranaivoson, Wilfrid Chrysante Solofoarisina}

Theoretical Physics Department, Institut National des Sciences et Techniques Nucléaires (INSTN-Madagascar), Antananarivo, Madagascar

\section{Email address:}

instn@moov.mg (Raoelina Andriambololona), raoelinasp@yahoo.fr (Raoelina Andriambololona), jacquelineraoelina@hotmail.com (Raoelina Andriambololona), tokhiniaina@gmail.com (R. T. Ranaivoson), s.wilfride@moov.mg (W. C. Solofoarisina),wilfridc_solofoarisina@yahoo.fr (W. C. Solofoarisina)

\section{To cite this article:}

Raoelina Andriambololona, Ravo Tokiniaina Ranaivoson, Wilfrid Chrysante Solofoarisina. Arithmetic and Matricial Calculation. Pure and Applied Mathematics Journal. Vol. 5, No. 3, 2016, pp. 82-86. doi: 10.11648/j.pamj.20160503.14

Received: April 29, 2016; Accepted: May 9, 2016; Published: May 25, 2016

\begin{abstract}
We present a study on written numeration and arithmetic using matricial formalism for the writing of numeral basis and number. The underlying idea is simple, it consists to consider the numeral representation of number as a matrix representation of an intrinsic number in a basis which represents the numeral system. Then the matrix calculation and linear algebra tools are extensively utilized to simplify arithmetic operations and to remove many inconsistencies existing in arithmetic. Owing to the adopted convention, four dispositions are obtained for the writing of number components according to the disposition in row matrix or column matrix and in decreasing or increasing order. The writing in line from Left handside to the Right handsideby increasing order (called LRi) is shown to be much more logical and coherent with the addition and the multiplication rules than the usual one which starts from the left handside to the right handside by decreasing order (LRd). In the LRi disposition, rules for the addition and multiplication of integers number are derived.
\end{abstract}

Keywords: Arithmetic, Matricial Calculation, Numeral System, Radix, Basis, Arithmetic Operations

\section{Introduction}

The problem of numeration concerns the writing and the designation of numbers [1], [2]. Numbers can be defined intrinsically without a dependence on the name to spell them: it is the number theory linked with the study of ordinals in the axiomatic theory of set of Zermelo and Fraenkel [3].

When a numeral system is given, a number is well defined, one can write it explicitly: it is the problem of writing numeration which is directly and logically linked to the used writing. The choice of numeral system $(2,3,5,6,8,10,16$, $32, .$.$) depends on many reasons (civilization, philosophy,...).$ For instance in a logics with two values (no, yes) it is convenient to use the binary system [4], [5].

The present work deals with the problem of writing numeration and arithmetic using matricial calculation and other tools from linear algebra [6], [7], [8]. This approach was introduced and developed by Raoelina Andriambololona [9]. In his works in 1987, he considered an analogy between the elements of a numeral system, the power $b^{s}$ of the radix $b$, and vector basis as defined in the framework of linear algebra. This approach led him to matrix representations of numeration basis and numbers and the use of matrix calculation in arithmetic. He showed that owing to some adopted convention, four dispositions are obtained for the writing of number components according to the disposition in row matrix or column matrix and in decreasing or increasing order. $\mathrm{He}$ remarked that former disposition convention may be fixed in order that the writing of the number is logic and consistent with the word writing.

Raoelina Andriambololona's first works on the use of matricial calculation in arithmetics contained also studies on reading numeration and designation of number [10]. He showed that three steps are to be followed: first, denomination of a basis vectors having positive and null powers are given. The existence of periodicity is to be noted and its value is $r=3$ for french, english, german, hindi, ... languages, $r=4$ for chinese language, $r=5$ for gujarath language and $r=6$ for malagasy language. It is possible to choose $r \leq 3$ for language having $r>3$ like malagasy one.

Second, negative power vector basis names are derived from those of positive power vector basis by means of suffix or prefix signifying "part of" put together to the positive power vector basis name. In french language: the suffix is "ième" (e.g. dixième), in english: the suffix is "th" (e.g. tenth), 
in german: the suffix is "stel" (e.g. zehnstel), in swahili: the prefix is" ya" (e.g. yakumi), in malagasy: the prefix is "ampaha" (e.g. ampahafolo, etc...).

And third, the enunciation of an entire or decimal numbers follows exactly its writing, starting first from the basis vector name followed by the numeral representing the number component on the basis vector, the name of following basis vector with the annexed numeral and related o the proceding one by means of addition.

Addition is expressed either by conjunction ("sy" in malagasy, "et" in french, "and" in english, "und" in german, "na" in swahili, ...) or by simple juxtaposition if there is no ambiguity with multiplication. Multiplication is expressed either by its signification in the language "in" or "im" according to the grammar -in malagasy, "fois" in french...) or by simple juxtaposition when there is no ambiguity with addition.

He showed that the approach he has proposed is logic, systematic, self-consistent and rational; disadvantages appearing in usual language are avoided. It is general and applicable to any language.

As previously mentioned, the present paper is based on the method introduced and developed by Raoelina Andriambololona to tackle the problem of writing numeration and arithmetic using matricial calculation [9]. Through the next sections, we show and develop this method with illustrative examples.

The four possible dispositions obtained using matrix representation for the writing of number components and numeration basis are studied explicitly. The advantages of the writing in line from Left handside to the Right handside by increasing order (LRi) is shown.

Study on addition and multiplication in the framework of the approach is also performed.

Fundamentally, Raoelina Andriambololona considered a number given in a numeral system as a row matrix representation of an intrinsic number. For instance, the number 2015 given in the decimal system is the row matrix representation [ [ $\left.\begin{array}{llll}2 & 0 & 1 & 5\end{array}\right]$ of an intrinsic number $a$ expressed in the matricial basis $B=\left[\begin{array}{l}10^{3} \\ 10^{2} \\ 10^{1} \\ 10^{0}\end{array}\right]$ such as

$$
a=\left[\begin{array}{llll}
2 & 0 & 1 & 5
\end{array}\right]\left[\begin{array}{l}
10^{3} \\
10^{2} \\
10^{1} \\
10^{0}
\end{array}\right]
$$

\section{Numeral Systems and Matricial Basis}

Let $b$ be a given positive integer, $b$ belonging to $\mathbb{N}$. The number $b$ is called "radix of the numeral system". Let us consider the sequence $b^{s}$ of the powers of $b$ for $s$ an integer number, $s$ belonging to $\mathbb{Z} . b^{s}$ is called an "element" of the "basis" $\left\{b^{s}\right\}$ using the language of linear algebra [6]. A number $a$ (integer or decimal) can be expanded in the basis $\left\{b^{s}\right\}$ as a linear combination of the basis vector $b^{s}$ $a=\sum_{s=s_{1}}^{s=s_{2}} a_{s} b^{s}=a_{s_{1}} b^{s_{1}}+a_{s_{1}+1} b^{s_{1}+1}+\cdots+a_{s_{2}-1} b^{s_{2}-1}+a_{s_{2}} b^{s_{2}}$

where the $a_{s}$, which are not null all, are integer numbers strictly less than $b . a_{s}$ is called the "component" of $a$ on the basis element $b^{s}$. The set of $\left[a_{s}\right]$ may be written as a row matrix or as a column matrix representing the number $a$ in the numeral system with radix $b$.

The uniqueness of $a_{s}$ is deduced from the uniqueness of the quotient and the remainder of the euclidian division by multiplying $a$ by the power $b^{s^{\prime}}$ such as all $s^{\prime \prime}$ of the power $b^{s^{\prime \prime}}$ appearing in the product $a_{s} b^{s^{\prime}}$ are positive or null (or simply by the linear independence of vectors basis).

Example: let us consider the number $a$ represented by the symbol " 2015 " in the decimal system (the radix is $b=$ 10): $a=(2015)_{10}$. We have

$$
a=5 \times 10^{0}+1 \times 10^{1}+0 \times 10^{2}+2 \times 10^{3}
$$

We can choose other numeral systems.

-For $b=2$ (binary system), we have for the same number $a$ $a=1 \times 2^{0}+1 \times 2^{1}+1 \times 2^{2}+1 \times 2^{3}+1 \times 2^{4}+0 \times 2^{5}$

$$
+1 \times 2^{6}+1 \times 2^{7}+1 \times 2^{8}+1 \times 2^{9}+1 \times 2^{10}
$$

So in the binary system, $a$ is represented by the symbol “11111011111”.

$$
a=(2015)_{10}=(11111011111)_{2}
$$

-For $b=3$ (trinary system), $a=2 \times 3^{0}+2 \times 3^{1}+1 \times 3^{2}+2 \times 3^{3}+0 \times 3^{4}+2 \times 3^{5}+2 \times 3^{6}$

So in the trinary system, $a$ is represented by the symbol "2202122":

$$
a=(2015)_{10}=(2202122)_{3}
$$

-For $b=8$ (octal system)

$$
a=7 \times 8^{0}+3 \times 8^{1}+7 \times 8^{2}+3 \times 8^{3}
$$

So in the octal system, $a$ is represented by the symbol “3737".

$$
a=(2015)_{10}=(3737)_{8}
$$

We can represent the basis $\left\{b^{s}\right\}$ as a row matrix or a column matrix following a determined order: decreasing or increasing one. This gives us four types of possibility for the writing of a number:

\subsection{Basis $\left\{b^{s}\right\}$ Written as a Column Matrix from Top (T) to the Bottom (B) by Decreasing (d) Order TBd}

The number $a$ is written as a row matrix from left (L) handside to the right handside $(\mathrm{R})$ by decreasing (d) order (LRd).This is the international notation.

$$
a=\sum_{s=s_{1}}^{s=s_{2}} a_{s} b^{s}=\left[\begin{array}{lllll}
a_{s_{2}} & a_{s_{2}-1} & \ldots \ldots & a_{s_{1}+1} & a_{s_{1}}
\end{array}\right]\left[\begin{array}{c}
b^{s_{2}} \\
b^{s_{2}-1} \\
. \\
\vdots \\
b^{s_{1}+1} \\
b^{s_{1}}
\end{array}\right]
$$


If we consider the example of $a=(2015)_{10}$, we have -For $b=10$ (decimal system)

$a=5 \times 10^{0}+1 \times 10^{1}+0 \times 10^{2}+2 \times 10^{3}=\left[\begin{array}{llll}2 & 0 & 1 & 5\end{array}\right]\left[\begin{array}{l}10^{3} \\ 10^{2} \\ 10^{1} \\ 10^{0}\end{array}\right]$

-For $b=8$ (octal system)

$a=7 \times 8^{0}+3 \times 8^{1}+7 \times 8^{2}+3 \times 8^{3}=\left[\begin{array}{llll}3 & 7 & 3 & 7\end{array}\right]\left[\begin{array}{l}8^{3} \\ 8^{2} \\ 8^{1} \\ 8^{0}\end{array}\right]$

The same number $(2015)_{10}$ is represent by $(3737)_{8}$ in octal system

\subsection{Basis $\left\{b^{s}\right\}$ Written as a Column Matrix from Top (T) to the Bottom (B) by Increasing (i) Order TBi}

The number $a$ is written as a row matrix from left $(\mathrm{L})$ handside to the right handside (R) by increasing (i) order LRi

$a=\sum_{s=s_{1}}^{s=s_{2}} a_{s} b^{s}=\left[\begin{array}{lllll}a_{s_{1}} & a_{s_{1}+1} & \ldots \ldots & a_{s_{2}-1} & a_{s_{2}}\end{array}\right]\left[\begin{array}{c}b^{s_{1}} \\ b^{s_{1}+1} \\ . \\ \vdots \\ b^{s_{2}-1} \\ b^{s_{2}}\end{array}\right]$

If we consider the example of $a=(2015)_{10}$, we have -For $b=10$ (decimal system)

$a=5 \times 10^{0}+1 \times 10^{1}+0 \times 10^{2}+2 \times 10^{3}=\left[\begin{array}{llll}5 & 1 & 0 & 2\end{array}\right]\left[\begin{array}{l}10^{0} \\ 10^{1} \\ 10^{2} \\ 10^{3}\end{array}\right]$

-For $b=8$ (octal system)

$$
\begin{aligned}
a=(2015)_{10} & =7 \times 8^{0}+3 \times 8^{1}+7 \times 8^{2}+3 \times 8^{3} \\
& =\left[\begin{array}{llll}
7 & 3 & 7 & 3
\end{array}\right]\left[\begin{array}{l}
8^{0} \\
8^{1} \\
8^{2} \\
8^{3}
\end{array}\right]
\end{aligned}
$$

\subsection{Basis $\left\{b^{s}\right\}$ Written as a Row Matrix from the Left $(L)$ Handisde to Right (R) Handside by Decreasing(d) Order LRd}

The number $a$ is written as a column matrix from the top (T) to the bottom (B) by decreasing (d) order TBd

$$
a=\sum_{s=s_{1}}^{s=s_{2}} a_{s} b^{s}=\left[\begin{array}{lllll}
b^{s_{2}} & b^{s_{2}-1} & \ldots \ldots & b^{s_{1}+1} & b^{s_{1}}
\end{array}\right]\left[\begin{array}{c}
a_{s_{2}} \\
a_{s_{2}-1} \\
\vdots \\
\vdots \\
a_{s_{1}+1} \\
a_{s_{1}}
\end{array}\right]
$$

If we consider the example of $a=(2015)_{10}$, we have -For $b=10$ (decimal system)

$$
a=5 \times 10^{0}+1 \times 10^{1}+0 \times 10^{2}+2 \times 10^{3}
$$

$$
=\left[\begin{array}{llll}
10^{3} & 10^{2} & 10^{1} & 10^{1}
\end{array}\right]\left[\begin{array}{l}
2 \\
0 \\
1 \\
5
\end{array}\right]
$$

-For $b=8$ (octal system)

$$
a=7 \times 8^{0}+3 \times 8^{1}+7 \times 8^{2}+3 \times 8^{3}=\left[\begin{array}{llll}
8^{3} & 8^{2} & 8^{1} & 8^{0}
\end{array}\right]\left[\begin{array}{l}
3 \\
7 \\
3 \\
7
\end{array}\right]
$$

2.4. Basis $\left\{b^{s}\right\}$ Written as a Row Matrix from the Left (L)

Handisde to Right (R) Handside by Increasing (I) Order LRi

The number $a$ is written as a column matrix from the top (T) to the bottom (B) by increasing (i) order TBi

$$
a=\sum_{s=s_{1}}^{s=s_{2}} a_{s} b^{s}=\left[\begin{array}{lllll}
b^{s_{1}} & b^{s_{1}+1} & \ldots \ldots & b^{s_{2}-1} & b^{s_{2}}
\end{array}\right]\left[\begin{array}{c}
a_{s_{1}} \\
a_{s_{1}-1} \\
\vdots \\
\vdots \\
a_{s_{2}-1} \\
a_{s_{2}}
\end{array}\right]
$$

If we consider the example of $a=(2015)_{10}$, we have -For $b=10$ (decimal system)

$$
a=5 \times 10^{0}+1 \times 10^{1}+0 \times 10^{2}+2 \times 10^{3}
$$

$$
=\left[\begin{array}{llll}
10^{0} & 10^{1} & 10^{2} & 10^{3}
\end{array}\right]\left[\begin{array}{l}
5 \\
1 \\
0 \\
2
\end{array}\right]
$$

$$
\begin{aligned}
& \text {-For } b=8 \text { (octal system) } \\
& \qquad \begin{array}{c}
a=7 \times 8^{0}+3 \times 8^{1}+7 \times 8^{2}+3 \times 8^{3} \\
=\left[\begin{array}{llll}
8^{0} & 8^{1} & 8^{2} & 8^{3}
\end{array}\right]\left[\begin{array}{l}
7 \\
3 \\
7 \\
3
\end{array}\right]
\end{array}
\end{aligned}
$$

The values of the $a_{s}$, strictly less than $b$ are the figure of the system. One can represent the figure by the letter of the writing (for example the case of the greek numeration) or by drawing (egyptian and babylonian numeration) or more currently by special sign [1], [2].

We have different systems according to the value of $b$, for instance: the binary system $(b=2)$, the ternary system $(b=3)$, the octal system $(b=8)$, the decimal system $(b=10)$, the duo-decimal system $(b=12)$, the sexagesimal system $(b=60)$ [4].

\section{Advantage of the Writing in Line from Left Handside to Right Handside by Increasing Order LRi}

In the paragraph 2, we have shown that there are four possibilities for the writing of the basis-matrix ( $\mathrm{TBd}, \mathrm{TBi}$, LRd, LRi). As a consequence, we have four possibilities for the writing of a number in the numeral system with radix $b$ (LRd, LRi, TBd, TBi) too. The currently adopted dispositions is the writing in line from the left handside to the right 
handside by decreasing order LRd.

Beginning from the principle that the writing of a number and the operations on the number must be logical and coherent with the normal writing i.e the writing in line, we have only two possibilities: LRd and LRi.

The international writing LRd is inconsistent with the habitual rules of addition, substraction and multiplication which are done from the right handside to the left handside by increasing order LRi while the division is done LRd.

It is obvious that one can not establish logical and consistent rules of operations (addition, multiplication) by beginning from the left handside to the right handside because of the reports and the remainder which are necessarily done by increasing order.

We are led to the examination of the LRi writing of number. We establish new rules for the addition and multiplication which are homogenious and consistent with the LRi dispositions. We may obtain rules for the cases of substraction and division which are consistent with the LRi disposition too. It is different from the current international rules by only on some few points in particular because of the writing by increasing order of number.

As shown in the relation (4) in the LRi writing of a number, the basis is a column matrix and the number is a row matrix

$$
a=\sum_{s=s_{1}}^{s=s_{2}} a_{s} b^{s}=\left[\begin{array}{lllll}
a_{s_{1}} & a_{s_{1}+1} & \ldots \ldots & a_{s_{2}-1} & a_{s_{2}}
\end{array}\right]\left[\begin{array}{c}
b^{s_{1}} \\
b^{s_{1}+1} \\
\vdots \\
\vdots \\
b^{s_{2}-1} \\
b^{s_{2}}
\end{array}\right]
$$

In particular we have for the number $b^{i}$

$$
b^{i}=\left[\begin{array}{lllll}
0 & 0 & \ldots 1 \ldots & 0 & 0
\end{array}\right]\left[\begin{array}{c}
0 \\
0 \\
\vdots \\
\cdot \\
b^{i} \\
\cdot \\
\dot{0} \\
0 \\
0
\end{array}\right]
$$

If we denote $W_{b}(a)$ the row matrix representing, in the LRi writing, the number $a$ and $B$ the column matrix representing the basis

$$
W_{b}(a)=\left[\begin{array}{lllll}
a_{s_{1}} & a_{s_{1}+1} & \ldots \ldots & a_{s_{2}-1} & a_{s_{2}}
\end{array}\right] B=\left[\begin{array}{c}
b^{s_{1}} \\
b^{s_{1}+1} \\
. \\
\vdots \\
b^{s_{2}-1} \\
b^{s_{2}}
\end{array}\right]
$$

the relation (3.1) gives

$$
a=W_{b}(a) B
$$

- The elements $a_{i}$ of the matrix $W_{b}(a)$ are null or less than $b$, they belong to the set $\{0,1, \ldots, b-1\}$. The numbers $0,1, \ldots, b-1$ are the figures of the numeration in the numeral system with radix $b$.
Given two integers numbers $a$ and $a^{\prime}$

$$
\begin{aligned}
& a=W_{b}(a) B \\
& a^{\prime}=W_{b}\left(a^{\prime}\right) B
\end{aligned}
$$

We look for the rules to be applied on $W_{b}(a)$ and $W_{b}\left(a^{\prime}\right)$ to obtain the sum

$$
a+a^{\prime}=W_{b}\left(a+a^{\prime}\right) B
$$

and the product

$$
a a^{\prime}=W_{b}\left(a a^{\prime}\right) B
$$

in the numeral system with radix $b$.

\section{Rules for the Addition of Two Numbers}

We have for two positive integers $a$ and $a^{\prime}$

$$
\begin{aligned}
& a=W_{b}(a) B=\sum_{i=0}^{n} a_{i} b^{i} \\
& a^{\prime}=W_{b}\left(a^{\prime}\right) B=\sum_{i=0}^{n^{\prime}} a_{i}^{\prime} b^{i}
\end{aligned}
$$

We can suppose $n^{\prime}>n$ with $a_{n}^{\prime} \neq 0, a_{i}=0$ for $n+1 \leq$ $i \leq n^{\prime}$, without loosing in generality.

$$
a+a^{\prime}=W_{b}\left(a+a^{\prime}\right) B=\sum_{i=0}^{n} a_{i} b^{i}+\sum_{i=0}^{n^{\prime}} a_{i}^{\prime} b^{i}=\sum_{i=0}^{n^{\prime \prime}} a_{i}^{\prime \prime} b^{i}
$$

The integer $n^{\prime \prime}$ must be chosen so that $a_{i}^{\prime \prime}<b$ for all $i$ and in particular for $i=n^{\prime}$. In fact we know that the elements $a_{i}^{\prime \prime}$ of the row matrix $W_{b}\left(a+a^{\prime}\right)$ must be null or strictly less than $b$ :

- If $a_{i}+a_{i}^{\prime}<b$, then we have $a_{i}^{\prime \prime}=a_{i}+a_{i}^{\prime}$ is the element at the $(i+1)^{t h}$ column (from the left handside) of the matrix $W_{b}\left(a+a^{\prime}\right)$

- If $a_{i}+a_{i}^{\prime}>b$, we perform the decompositions

$$
\begin{gathered}
a_{i}^{\prime \prime}=a_{i s} b^{s}+\cdots+a_{i 1} b^{1}+a_{i 0} b^{0} \text { with } a_{i s}<b \\
a_{i}^{\prime \prime} b^{i}=a_{i s} b^{s+i}+\cdots+a_{i 1} b^{1+i}+a_{i 0} b^{i}
\end{gathered}
$$

without summation on $i$. The element at the $(i+1)^{\text {th }}$ column from the left handside of $W_{b}\left(a+a^{\prime}\right)$ is $a_{i}^{\prime \prime}=a_{i 0}$ while the remainders $a_{i 1}, a_{i 2}, \ldots$ are to be brought respectively at the $(i+2)^{t h},(i+3)^{t h}, \ldots(i+s+1)^{t h}$ column (from the left) of $W_{b}\left(a+a^{\prime}\right)$. Then, the addition is done from the left handside to the right handside by increasing order LRi.

The 3 by 3 terms grouping is also done LR but not RL, that is consistent with the writing.

The rule may be easily extended to the sum of many numbers

\section{Rules for the Multiplication}

Let $a$ and $a^{\prime}$ be two positive integers

$$
\begin{aligned}
& a=W_{b}(a) B=\sum_{i=1}^{n} a_{i} b^{i} \\
& a^{\prime}=W_{b}\left(a^{\prime}\right) B=\sum_{i=1}^{n^{\prime}} a_{i}^{\prime} b^{i}
\end{aligned}
$$

Let be 


$$
a a^{\prime}=W_{b}\left(a a^{\prime}\right) B=\sum_{i=0}^{n^{\prime \prime}}\left(a a^{\prime}\right)_{i} b^{i}
$$

For the explicit calculation, we proceed as for the case of the addition in respecting the places

$$
\begin{gathered}
a a^{\prime}=a_{0} b^{0}\left(a_{0}^{\prime} b^{0}+a_{1}^{\prime} b^{1}+\cdots a_{n}^{\prime} b^{n}\right) \\
+a_{1} b^{1}\left(a_{0}^{\prime} b^{0}+a_{1}^{\prime} b^{1}+\cdots a_{n}^{\prime} b^{n}\right)+\cdots \\
=a_{0} a_{0}^{\prime} b^{0}+\sum_{i+j=0}^{1}\left(a_{i} a_{k}^{\prime}\right) b^{1}+\sum_{i+j=0}^{2}\left(a_{i} a_{k}^{\prime}\right) b^{2}+\cdots
\end{gathered}
$$

In the calculation of the component $\left(a a^{\prime}\right)_{i}$, of the product $a a^{\prime}$, which must be strictly less than $b$, we must bring the remainder in the partial sum.

The division of a number $a^{\prime \prime}$ by a number $a$ is the research of the number $a^{\prime}$ such as $a^{\prime \prime}=a a^{\prime}$. It is the inverse operation of multiplication. We can also use the inverse of the multiplication operation and establish the LRi procedure. The rules can be obtained by analogy with the multiplication rules.

\section{Conclusion}

The above results show that the use of matrix formalism and matrix calculation in arithmetic may be considered as an interesting approach. It permits, among other results, to have new insights concerning writing numeration and arithmetic operations. As an example, this approach shows that the writing in line from Left handside to the Right handside by increasing order LRi is the most logical and the most consistent with the arithmetic operations rules.

This approach may have many interesting uses in all areas of the arithmetics and its applications. As examples, we have already studied the problem of numeral system change [11] and the implementation of arithmetic operations in the framework of computer science. [12]

\section{References}

[1] Georges Ifrah, David Bellos, E. F. Harding, Sophie Wood, Ian Monk, "The Universal History of Numbers: From Prehistory to the Invention of the Computer", John Wiley \& Sons, New York, 1999.

[2] Stephen Chrisomalis, "Numerical Notation: A Comparative History", Cambridge University Press, 2010.

[3] Encyclopédie Universalis, "Théorie axiomatique des ensembles. Chapitre 4", Vol. 10 pages 66, 1968.

[4] Anton Glaser, "History of binary and other nondecimal numeration", Tomash, 1971.

[5] M. Morris Mano, Charles Kime. "Logic and computer design fundamentals." (4th ed.). Pearson, 2014.

[6] Raoelina Andriambololona, "Algèbre linéaire et multilinéaire", Collection LIRA, INSTN-Madagascar, Antananarivo, Madagascar, 1986.

[7] Anton Howard, Chris Rorres, "Elementary Linear Algebra" (10th ed.), John Wiley \& Sons, 2010.

[8] William C. Brown "Matrices and vector spaces", New York, NY: Marcel Dekker, 1991.

[9] Raoelina Andriambololona, "Théorie générale des numérations écrite et parlée. II Utilisation du calcul matriciel en arithmétique. Nouvelle proposition d'écriture, d'énoncé des règles d'addition et de multiplication des nombres.". Bull. Acad.Malg LXV/1-2, Antananarivo, Madagascar, 1987.

[10] Raoelina Andriambololona, "Théorie générale des numérations écrite et parlée”. Bull. Acad. Malg. LXIV./1-2, Antananarivo, Madagascar, 1986.

[11] Raoelina Andriambololona, "Théorie générale des numérations écrite et parlée. II- Utilisation du calcul matriciel en arithmétique. Application au changement de bases de numération. Bull. Acad. Malg. LXV./1-2, Antananarivo, Madagascar”, 1987 (1989).

[12] Raoelina Andriambololona, Hanitriarivo Rakotoson "Mpikajy elekronika sy siantifika mampiasa ny fomba fanisana Malagasy (Electronic and scientific calculator based on malagasy counting method)", communication at the Academie Malgache, Antananarivo Madagascar, 05 June 2008. 